TRANSACTIONS OF THE

AMERICAN MATHEMATICAL SOCIETY

Volume 353, Number 6, Pages 2371-2390

S 0002-9947(01)02517-X

Article electronically published on February 13, 2001

\title{
ON THE COMPUTATION OF STABILIZED TENSOR FUNCTORS AND THE RELATIVE ALGEBRAIC $K$-THEORY OF DUAL NUMBERS
}

\author{
RANDY MCCARTHY
}

\begin{abstract}
We compute the stabilization of functors from exact categories to abelian groups derived from $n$-fold tensor products. Rationally, this gives a new computation for the relative algebraic $K$-theory of dual numbers.
\end{abstract}

\section{INTRODUCTION}

In [8], T. Goodwillie computed the relative algebraic $K$-theory of dual numbers rationally and used this to show that a suitable trace map to cyclic homology is a rational equivalence on relative theories for nilpotent extensions. In [5], a new model for the relative algebraic $K$-theory of dual numbers was introduced and used to show the equivalence of stable $K$-theory and topological Hochschild homologywhich was shown in [16] to be equivalent to Mac Lane homology [10]. One of our goals in this paper is to give a new computation of Goodwillie's result by exploiting the model from [5]. To do this, one is lead to compute the stabilization of various functors from exact categories to abelian groups, as introduced in [14. Essentially, given a functor $F$ from exact categories to abelian groups, its stabilization is defined as: $\quad F^{s t}=\lim _{n \rightarrow \infty} F\left(S^{(n)}\right)[-n]$, where $S^{(n)}$ is Waldhausen's $S$ construction for exact categories ([17]) iterated $n$ times. This can be thought of as a generalization of the bar construction for abelian groups, and hence this stabilization is a direct transliteration of the Dold-Puppe stable derived functors ([4]) to the setting of exact categories. Our method of computation for the stabilized functors is first to relate them to a stabilized version of the cohomology of small categories in the sense of [2] and then to relate this to Mac Lane homology much in the manner of [9].

There is a functor $\mathcal{S}_{*}$ from exact categories to categories of exact categories such that $\mathcal{S}_{\mathcal{A}}$ is the smallest subcategory of all exact categories containing $\mathcal{A}$ which is closed under isomorphisms and taking Waldhausen's $S$. construction (see section 0 for more details). Let $R$ be a ring and let $M$ be an $R$-bimodule. Let $\mathcal{M}$ be the category of all (right) $R$-modules and let $\mathcal{P}$ be the full category of finitely generated projective $R$-modules. We write $\mathcal{S}_{I}$ for $\mathcal{S}_{*}$ of the exact inclusion functor $I: \mathcal{P} \longrightarrow \mathcal{M}$, and similarly $\mathcal{S}_{M}$ for $\mathcal{S}_{*}$ of the exact functor $\star \otimes_{R} M$ from $\mathcal{P}$ to $\mathcal{M}$. Let $Q_{*}(R)$ be Mac Lane's $Q$-construction ([10]).

Received by the editors February 14, 1996 and, in revised form, January 19, 1999.

1991 Mathematics Subject Classification. Primary 19D55, 55U99, 18G99.

Research supported in part by National Science Foundation grant DMS 94-15615 and a Sloan Fellowship. 
Theorem (4.1). Let $G$ be the functor from $\mathcal{S}_{\mathcal{P}}$ to abelian groups defined by

$$
G(\mathcal{A})=\bigoplus_{A \in \mathcal{A}} \bigotimes_{i=1}^{n} \operatorname{Hom}_{\mathcal{S}_{I}(\mathcal{A})}\left(\mathcal{S}_{I}(A), \mathcal{S}_{M}(A)\right) .
$$

Then $G^{\text {st }}$ is completely determined by its value at $\mathcal{P}$, and

$$
G^{s t}(\mathcal{P}) \sim_{\Sigma_{n}} \mathbf{Z}\left[\Sigma_{n}\right] \otimes_{\mathbf{Z}\left[C_{n}\right]} H H\left(Q_{*}(R)^{\otimes n} ; M_{\tau}^{\otimes n}\right),
$$

where $H H$ is the Hochschild homology complex for $Q_{*}(R)^{\otimes n}$ acting on the bimodule by

$$
\begin{gathered}
\left(m_{1} \otimes \cdots \otimes m_{n}\right) *\left(q_{1} \otimes \cdots \otimes q_{n}\right)=\left(m_{1} q_{1} \otimes m_{2} q_{2} \otimes \cdots \otimes m_{n} q_{n}\right), \\
\left(q_{1} \otimes \cdots \otimes q_{n}\right) *\left(m_{1} \otimes \cdots \otimes m_{n}\right)=\left(q_{n} m_{1} \otimes q_{1} m_{2} \otimes \cdots \otimes q_{n-1} m_{n}\right),
\end{gathered}
$$

and $C_{n}$ is the cyclic group of $n$ elements which acts by cyclic permutations - the equivalence is weakly $\Sigma_{n}$-equivariant.

The paper is organized as follows. In section 0 we recall some terminology from [17 and establish some notation. In section 1 we show how to reduce the computation of relative algebraic $K$-theory of dual numbers rationally to that of the stabilization of functors like $G$ in the above proposition. In section 2 we generalize a result of [7] to rewrite these in terms of a suitably stabilized cohomology of small categories. In section 3 we further reduce these models to appropriate (no longer stabilized) cohomology of small categories. In section 4 we reinterpret these results in terms of Mac Lane homology following the ideas of $[9]$.

\section{Preliminaries-MAKing a FUnCtor AdDitive By STABilization}

In this section we recall the definition of the $S$ construction from [17] and establish some notation. We then recall the notion of stabilization for functors from exact categories to chain complexes as introduced in [14].

For $q \in \mathbf{N}$, let $[q]$ denote the poset $\{0<\cdots<q\}$, which we will often view as a category. For $\mathcal{C}$ (small) and $\mathcal{D}$ categories, let $\operatorname{Fun}(\mathcal{C}, \mathcal{D})$ be the category of functors from $\mathcal{C}$ to $\mathcal{D}$ and morphisms the natural transformations of these. For $\mathcal{D}$ a category, let the arrow category, $\operatorname{Ar} \mathcal{D}$, be $\operatorname{Fun}([1], \mathcal{D})$.

Let $\mathcal{E}$ be an exact category considered as a category with cofibrations ([17], first few lines) by setting the subcategory of cofibrations to be the admissible monomorphisms. We let $\operatorname{Ex}(\operatorname{Ar}[q], \mathcal{E})$ be the full subcategory of $\operatorname{Fun}(\operatorname{Ar}[q], \mathcal{E})$ whose objects are the functors $F$ such that $F(j \rightarrow j)=*$ and, for every triple $i \leq j \leq k$ in $[q]$,

$$
F(i \rightarrow j) \rightarrow F(i \rightarrow k) \rightarrow F(j \rightarrow k)
$$

is a short exact sequence. Setting $S_{[q]} \mathcal{E}=\operatorname{Ex}(\operatorname{Ar}[q], \mathcal{E})$, we obtain a simplicial exact category, and we write $S \mathcal{E}$ for both this simplicial exact category and the associated simplicial set we obtain by taking the set of objects degreewise. We can iterate the $S$ construction, and we write $\mathbf{K C}$ for the algebraic $K$-theory (pre-)spectrum of $\mathcal{C}$, $\mathbf{K C}=\left\{\left|S^{(n)} \mathcal{C}\right|\right\}_{n \geq 0}$ (with structure maps constructed by the natural isomorphism $\left.\mathcal{C} \cong S_{1} \mathcal{C}\right)$.

Conventions: We will make no notational distinction between a (multi-dimensional) simplicial abelian group and its associated (multi-dimensional) chain complex. By a chain complex we will always mean a complex which is bounded below and homologically trivial in negative dimensions (i.e. connective). Given a multi-dimensional 
chain complex, we will consider it as a chain complex by taking Tot (using products). What follows has standard generalizations to various categories with cofibrations; but as these extensions are "straightforward" for the expert and do little more than cloud the essential ideas, we will keep our attention to exact categories.

Let $F$ be any functor from (small) linear categories (with a distinguished zero object 0 ) to chain complexes. It will be convenient for us to assume further that $F$ is reduced. That is, that $F(0)=0$. We will always think of an abelian group as a chain complex concentrated in dimension 0 .

We note that we are not assuming that $F$ takes naturally equivalent linear categories to homotopic chain complexes. If $F$ does this, we will say that $F$ is an equivalence functor. If $G$. is any functor from some category $\mathcal{C}$ of categories to (small) simplicial linear categories, then we can of course compose functors to obtain a new functor $F G$. from $\mathcal{C}$ to simplicial chain complexes, which we once again consider as a functor to chain complexes by taking Tot. By definition, if $\mathcal{A}_{*}$ is a simplicial (small) linear category, then $F \mathcal{A}_{*}$ is the simplicial chain complex obtained by applying $F$ degreewise.

We will say that $F$ is product preserving if for any two (small) exact categories $\mathcal{A}$ and $\mathcal{B}$, the natural projection map $\rho$ of simplicial abelian groups $F(\mathcal{A} \times \mathcal{B})$ to $F(\mathcal{A}) \times F(\mathcal{B})$ is a homotopy equivalence. We will say that $F$ is a $p$-product functor if $F$ preserves products in a range $0 \leq i \leq p$ (that is, $\pi_{i}(\rho)$ is an isomorphism for all $0 \leq i \leq p$ ). By the proof of additivity found in [12], for any $F$

$$
F S . S_{2} \mathcal{C} \stackrel{d_{0} \times d_{2}}{\longrightarrow} F S .(\mathcal{C} \times \mathcal{C})
$$

is an equivalence. If $F$ is a $p$-product functor, then the natural map $F\left(S S_{2}\right) \stackrel{d_{0} \times d_{2}}{\longrightarrow}$ $F S \times F S$ is an equivalence in a $p$-range. If $F S_{2} \longrightarrow F \times F$ is an equivalence in a $p$ range, then we say that $F$ is additive in a $p$ range.

Lemma (1.5 of [14]). For any $n \geq 1$, the functor $F S^{(n)}$ is a reduced equivalence functor which is a $2 n-1$ product functor and additive in a $2 n-1$ range.

For $X$ a chain complex, we let $X[z]$ be the new chain complex with $X[z]_{n}=X_{n-z}$ and $\partial[z]_{n}=\partial_{n-z}$.

Definition (0.1). For any exact category, we define

$$
F_{*}^{s t}(\mathcal{A})=\lim _{n \rightarrow \infty} F S^{(n)} \mathcal{A}[-n],
$$

which is a natural additive equivalence functor. We let $\alpha: F \rightarrow F^{s t}$ be the natural transformation obtained by the structure maps for the limit system. By lemma 1.7 of [14], $F$ is an additive functor (additive in an $\infty$-range) if and only if $F \rightarrow F^{s t}$ is an equivalence for all exact categories.

Examples. 1) Let $\mathbf{Z}$ be the functor which takes a (small) category $\mathcal{C}$ to the reduced free abelian group generated by the set of objects of $\mathcal{C}$. That is,

$$
\mathbf{Z}(\mathcal{C})=\text { cokernel }[\mathbf{Z}[0] \rightarrow \mathbf{Z}[\operatorname{Obj}(\mathcal{C})]]
$$

Then, by [14], $\mathbf{Z}^{\text {st }}$ is the stable homology functor and

$$
H_{*}\left(\mathbf{Z}^{s t}(\mathcal{C})\right)=H_{*}(\mathbf{K}(\mathcal{C}))=\pi_{*}(\mathbf{K}(\mathcal{C}) \wedge \mathbf{H Z}) .
$$


2) Let $\mathcal{A}$ be an exact category. Let Hom be the functor defined by

$$
\operatorname{Hom}(\mathcal{A})=\bigoplus_{A \in \mathcal{A}} \operatorname{Hom}_{\mathcal{A}}(A, A)
$$

Then, by [6],

$$
\operatorname{Hom}^{\text {st }}(\mathcal{A})=T H H(\mathcal{A}),
$$

where $T H H$ is the topological Hochschild homology of $\mathcal{A}$.

If $F$ is a functor defined on a subcategory $\mathcal{S}$ of all exact categories, then in order for $F^{s t}$ to still be defined we simply need that if $\mathcal{A} \in \mathcal{S}$ then $S . \mathcal{A}$ is a simplicial $\mathcal{S}$-object. In what follows we will need to restrict ourselves to functors defined on such subcategories of all exact categories. In particular, if $\mathcal{A}$ is an exact category we let $\mathcal{S}_{\mathcal{A}}$ be the smallest subcategory which contains $\mathcal{A}$, is closed under taking $S . \mathcal{A}$ (that is, $S . \mathcal{A}$ is a simplicial $\mathcal{S}_{\mathcal{A}}$ object) and is closed under isomorphisms (if an exact category $\mathcal{E}$ is isomorphic to an exact category in $\mathcal{S}_{\mathcal{A}}$ then it is in $\mathcal{S}_{\mathcal{A}}$ ). The category $\mathcal{S}_{\mathcal{A}}$ is skeletally small and is equivalent to the category with objects $S_{n_{1}} S_{n_{2}} \cdots S_{n_{t}} \mathcal{A}$ for all $t \geq 0$ and finite sequences $\left(n_{1}, \ldots, n_{t}\right)$ of non-negative integers with morphisms those determined by the $S$. construction. In this way we see that for any exact functor $F: \mathcal{A} \longrightarrow \mathcal{B}$ we get a functor $\mathcal{S}_{F}: \mathcal{S}_{\mathcal{A}} \longrightarrow \mathcal{S}_{\mathcal{B}}$ determined by $S_{n_{1}} S_{n_{2}} \ldots S_{n_{t}} F$ for each finite sequence $\left(n_{1}, \ldots, n_{t}\right)$ of non-negative integers. Thus, $\mathcal{S}_{*}$ is a functor from the category of exact categories to (skeletally small) categories of exact categories. For $F$ an exact functor from $\mathcal{A}$ to $\mathcal{B}, \mathcal{E} \in \mathcal{S}_{\mathcal{A}}$ and $E$ an object of $\mathcal{E}$, we abuse notation as follows: $\mathcal{S}_{F}$ is a functor from $\mathcal{S}_{\mathcal{A}}$ to $\mathcal{S}_{\mathcal{B}}$ which produces a functor $\left.\mathcal{S}_{F}\right|_{\mathcal{E}}$ from $\mathcal{E}$ to $S_{F}(\mathcal{E})$, and we set

$$
S_{F}(E)=\left.S_{F}\right|_{\mathcal{E}}(E) \in S_{F}(\mathcal{E}) .
$$

Example. 3) Let $R$ be a ring and let $M$ be an $R$-bimodule. Let $\mathcal{M}$ be the category of all (right) $R$-modules and let $\mathcal{P}$ be the full category of finitely generated projective $R$-modules. Let $I$ be the exact inclusion functor $I: \mathcal{P} \longrightarrow \mathcal{M}$ and let $M$ be the exact functor $\star \otimes_{R} M$ from $\mathcal{P}$ to $\mathcal{M}$. Let $\mathbf{M}$ be the functor from $\mathcal{S}_{\mathcal{P}}$ to abelian groups defined by

$$
\mathbf{M}(\mathcal{A})=\bigoplus_{A \in \mathcal{A}} \operatorname{Hom}_{\mathcal{S}_{I}(\mathcal{A})}\left(\mathcal{S}_{I}(A), \mathcal{S}_{M}(A)\right) .
$$

By section 2 of [5],

$$
\mathbf{M}^{s t}(\mathcal{A})=T H H(R ; M),
$$

where $\operatorname{THH}(R, M)$ is the topological Hochschild homology of the ring spectrum HR with coefficients in the bimodule HM.

\section{On the computation of $\tilde{K}(R \oplus M)_{\mathbf{Q}}$}

Definition. Following [5], for $R$ a ring, $M$ an $R$-bimodule and $X$ a space (= finite pointed simplicial set) we define $\tilde{\mathbf{K}}(R, \tilde{M}[X])$ to be the connective (pre-)spectrum:

$$
\tilde{K}(R, \tilde{M}[X])(n)=\left|[p] \times[q] \mapsto \bigvee_{\bar{P} \in S_{q}^{(n)} \mathcal{P}} \operatorname{Hom}_{S_{q}^{(n)} \mathcal{M}}\left(\bar{P}, \bar{P} \otimes_{R} \tilde{M}\left[X_{p}\right]\right)\right|,
$$

where $\mathcal{P}$ is the exact category of finitely generated $R$-modules, $\mathcal{M}$ the exact category of all $R$-modules and $\tilde{M}\left[X_{p}\right]=\bigoplus_{X_{p} \text {-basept }} M$. By section 4 of [5], if we let $R \oplus M$ 
be the ring with multiplication defined by $(r, m)\left(r^{\prime}, m^{\prime}\right)=\left(r r^{\prime}, r m^{\prime}+m r^{\prime}\right)$, then $\mathbf{K}(R \oplus M)$ is naturally equivalent to $\mathbf{K}(R) \times \tilde{\mathbf{K}}\left(R ; \tilde{M}\left[S^{1}\right]\right)$.

For any connective spectrum (of CW-type), the Hurewicz map produces an isomorphism from the rational homotopy groups of the spectrum to its rational homology groups (see for example page 203 of [1]). Thus,

$$
\begin{aligned}
\pi_{n}(\tilde{\mathbf{K}}(R \oplus M)) \otimes_{\mathbf{z}} \mathbf{Q} \cong \pi_{n} \tilde{\mathbf{K}}\left(R ; \tilde{M}\left[S^{1}\right]\right) \otimes_{\mathbf{Z}} \mathbf{Q} \\
\quad \stackrel{\cong}{\longrightarrow} \lim _{k \rightarrow \infty} H_{n+k}\left(\left|[p] \times[q] \mapsto \bigvee_{\bar{P} \in S_{q}^{(k)} \mathcal{P}} H_{o m}{ }_{S_{q}^{(k)} \mathcal{M}}\left(\bar{P}, \bar{P} \otimes_{R} \tilde{M}\left[X_{p}\right]\right)\right| ; \mathbf{Q}\right) .
\end{aligned}
$$

In general, for any (simplicial) bimodule $M$ and abelian group $G$, we obtain

$$
\begin{aligned}
H_{n}(\tilde{\mathbf{K}}(R ; M) ; G) & \simeq \lim _{k \rightarrow \infty} \pi_{n+k}\left|[q] \mapsto \tilde{G}\left[\bigvee_{\bar{P} \in S_{q}^{(k)} \mathcal{P}} \operatorname{Hom}_{S_{q}^{(k)} \mathcal{M}}\left(\bar{P}, \bar{P} \otimes_{R} M\right)\right]\right| \\
& \simeq \lim _{k \rightarrow \infty} \pi_{n+k}\left|[q] \mapsto \bigoplus_{\bar{P} \in S_{q}^{(k)} \mathcal{P}} \tilde{G}\left[\operatorname{Hom}_{S_{q}^{(k)} \mathcal{M}}\left(\bar{P}, \bar{P} \otimes_{R} M\right)\right]\right| \\
& =\tilde{G}[M]^{s t}(\mathcal{P}),
\end{aligned}
$$

where $\tilde{G}[M]$ is the functor from $\mathcal{S}_{\mathcal{P}}$ to simplicial abelian groups defined by

$$
\tilde{G}[M](\mathcal{A})=\bigoplus_{A \in \mathcal{A}} \tilde{G}\left[\operatorname{Hom}_{\mathcal{S}_{I}(\mathcal{A})}\left(\mathcal{S}_{I}(A), \mathcal{S}_{M}(A)\right)\right]
$$

and $G[M]^{s t}$ is defined as in 0.1. Putting these remarks together, we obtain the following proposition.

Proposition (1.1). For any ring $R$ and $R$-bimodule $M$,

$$
\pi_{n}(\tilde{\mathbf{K}}(R \oplus M)) \otimes_{\mathbf{Z}} \mathbf{Q} \cong H_{n}\left(\tilde{\mathbf{Q}}[B \cdot M]^{s t}(\mathcal{P})\right),
$$

where B.M $=\tilde{M}\left[S^{1}\right]$ is the usual bar construction for the abelian group $M$ considered as a simplicial $R$-bimodule.

To examine $\tilde{\mathbf{Q}}[B . M]$, we first recall some well known results. If $G$ is an abelian group, we let $p^{n}: G \longrightarrow G^{\otimes n}$ be the map of pointed sets defined by

$$
p^{n}(g)=\overbrace{g \otimes \cdots \otimes g}^{n \text { times }} .
$$

We abuse notation, and also write $p^{n}$ for the composed map

$$
G \stackrel{p^{n}}{\longrightarrow} G^{\otimes n} \stackrel{\rho}{\longrightarrow} G_{\mathbf{Q}} \otimes \cdots \otimes G_{\mathbf{Q}} \otimes_{\mathbf{Q}\left[\Sigma_{n}\right]} \mathbf{Q}=S^{n}\left(G_{\mathbf{Q}}\right),
$$

where $G_{\mathbf{Q}}=G \otimes \mathbf{z} \mathbf{Q}, \rho$ is the natural map and $\Sigma_{n}$ acts on the tensor product by permuting factors. Extending by linearity, we obtain a natural transformation of functors from abelian groups to rational vector spaces

$$
\tilde{\mathbf{Q}}[G] \stackrel{p}{\longrightarrow} \prod_{n \in \mathbf{N}} S^{n}\left(G_{\mathbf{Q}}\right) .
$$

We extend $p$ to simplicial abelian groups by evaluating everything degreewise. 
In general, the map $p$ is not an isomorphism, but it is an equivalence for 0 connected simplicial abelian groups. One can see this as follows. First we recall that (see for example theorem V.7.6 of [18])

$$
H_{*}(K(\mathbf{Z} / m \mathbf{Z} ; 1) ; \mathbf{Q})= \begin{cases}\mathbf{Q}, & i=0, \\ \mathbf{Q}, & i=1 \text { and } m=0, \\ 0, & \text { otherwise }\end{cases}
$$

By the Künneth theorem, this implies that

$$
H_{n}(K(G ; 1), \mathbf{Q})=\left(G_{\mathbf{Q}} \otimes \cdots \otimes G_{\mathbf{Q}}\right)^{s g n} \otimes_{\mathbf{Q}\left[\Sigma_{n}\right]} \mathbf{Q}=\bigwedge^{n}\left(G_{\mathbf{Q}}\right),
$$

where ( $)^{\text {sgn }}$ indicates we are now taking the $\Sigma_{n}$ action with signs and so $\bigwedge^{n}$ is the $n$-th exterior power. Now, $S^{n}\left(B . G_{\mathbf{Q}}\right)$ is simply $B^{n} G_{\mathbf{Q}}^{\otimes n} / \Sigma_{n}$, which (because the action of $\Sigma_{n}$ on the deloopings gives a signed action on the homotopy groups) is simply $B^{n}\left(\left(G_{\mathbf{Q}}^{\otimes n}\right)^{s g n} / \Sigma_{n}\right)$ (we are over $\mathbf{Q}$ ), and hence the result (after checking that the given transformation does indeed provide the correct map).

Corollary (1.2). Putting together the above remarks, we see that

$$
(\tilde{\mathbf{Q}}[B \cdot M])^{s t} \stackrel{\simeq}{\longrightarrow} \bigoplus_{n=1}^{\infty}\left(S^{n}\left[B \cdot M_{\mathbf{Q}}\right]\right)^{s t} .
$$

Next, we recall that since we are working over the rationals, a $\Sigma_{n}$ equivariant map which is also a weak equivalence is a weak equivalence on the map of orbits (since $\left|\Sigma_{n}\right|=n$ ! is invertible). Let $T^{n}(M)$ be the functor from $\mathcal{S}_{\mathcal{P}}$ to $\mathbf{Z}\left[\Sigma_{n}\right]$-modules defined by

$$
T^{n}(M)(\mathcal{A})=\bigoplus_{A \in \mathcal{A}} \bigotimes_{i=1}^{n} \operatorname{Hom}_{\mathcal{S}_{I}(\mathcal{A})}\left(\mathcal{S}_{I}(A), \mathcal{S}_{M}(A)\right)
$$

The following is a special case of the more general result in (4.1):

Proposition (1.3). Let $\mathbf{Q} \subseteq R$. Then

$$
T^{n}(B . M)^{s t}(\mathcal{P}) \sim_{\mathbf{Q}\left[\Sigma_{n}\right]} \mathbf{Q}\left[\Sigma_{n}\right] \otimes_{\mathbf{Q}\left[C_{n}\right]} H H\left(R^{\otimes n} ; B . M_{\tau}^{\otimes n}\right),
$$

where $\mathrm{HH}$ is the Hochschild homology and $\tau$ indicates the cyclic twisted action of $R^{\otimes n}$ on $X^{\otimes n}$ from the introduction. The cyclic group of order $n, C_{n}$, acts by permuting tensor factors. Hence

$$
\left.S^{n}(B \cdot M)^{s t}(\mathcal{P}) \simeq H H\left(R^{\otimes n} ; B \cdot M_{\tau}^{\otimes n}\right)\right] / C_{n}
$$

and

$$
\left.\frac{K(R \oplus M)}{K(R)} \simeq \mathbf{Q} \bigoplus_{n=1}^{\infty} H H\left(R^{\otimes n} ; B \cdot M_{\tau}^{\otimes n}\right)\right] / C_{n} .
$$

Remark. Using the techniques of [13] and the explicit maps used to obtain the above result, it is straightforward to show that the trace map from algebraic $K$-theory to negative homology used in [8] produces the needed isomorphism on relative theories after tensoring with the rationals. Since our objective here is to study the stabilized tensor functors, we will only give a brief outline below of how this can be done, and leave further details to the interested reader. 
Aside (1.4). On the rational equivalence of relative algebraic K-theory and relative negative cyclic homology.

The natural ring map $R \longrightarrow R \oplus M$ (taking $r$ to $(r, 0)$ ) produces a natural exact functor $\epsilon_{M}: \mathcal{P}_{R} \longrightarrow \mathcal{P}_{R \oplus M}$. By page 218 of [15] we have a natural transformation $\Phi$ of functors from $\mathcal{S}_{P} \times R$-Mod- $R$ to cyclic $\mathbf{Q}$-modules

$$
\Phi: \tilde{\mathbf{Q}}\left[N^{c y} *\right](\star) \longrightarrow H H\left(\mathcal{S}_{\epsilon_{*}} \star\right) .
$$

One always has a natural simplicial map $\rho$ from $B . M$ to $N^{c y} M$ defined by sending $\left(m_{1}, \ldots, m_{n}\right)$ to $\left(-\left(\Sigma_{i} m_{i}\right), m_{1}, \ldots, m_{n}\right)$, and the following diagram commutes (section 4 of [15]):

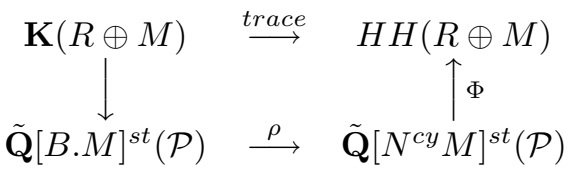

(the left vertical map is the composite of the equivalence from [5] with the Hurewicz map). In general, one can decompose $H H(R \oplus M)$ as the direct sum of cyclic abelian groups $\bigoplus_{i=0}^{\infty} H H^{[i]}(R \mid M)$, where $H H^{[i]}(R \mid M)_{[p]}$ is the submodule of $H H(R \oplus M)_{[p]}$ determined by sums of tensors $\left(x_{0} \otimes \cdots \otimes x_{n}\right)$ with exactly $i$ of the $x_{j}$ 's in $M$. One can extend this definition to $H N^{[i]}\left(\mathcal{S}_{\epsilon_{*} \star}\right)$ as a functor from $\mathcal{S}_{P}$ to (unbounded) chain complexes which is once again additive. For each $n \geq 0$ we obtain a factorization (up to equivalence)

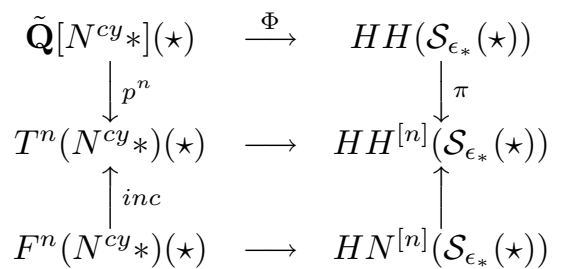

$\left(p^{n}\right.$ takes $[m]$ to $\left.m \otimes \cdots \otimes m\right)$. In the above diagram, $F^{n}=\left(\otimes^{n}\right)^{\Sigma_{n}}$, where the $\Sigma_{n}$ action is given by permuting tensor factors and the map inc is given by the inclusion $F^{n} \longrightarrow T^{n}$. Since we are working over $\mathbf{Q}$, the norm map from $S^{n}$ to $F^{n}$ is an equivalence which corresponds to the map from cyclic homology (orbits) to negative homology (fixed points), being an equivalence in this situation. By looking carefully at the computation for $H N(R \oplus M)$ in [8], one sees that the composite map $F^{n}(B . *)(\star)^{s t} \stackrel{\rho}{\longrightarrow} H N^{[n]}\left(S_{\epsilon_{*}} \star\right)^{s t} \stackrel{\simeq}{\longleftarrow} H N^{[n]}\left(S_{\epsilon_{*}} \star\right)$ is a rational equivalence, and hence the lift of the trace map to negative cyclic homology is rationally a relative equivalence for split square zero ring extensions.

This ends aside 1.4.

\section{A RELATion BETWEen STABILIZED FUnCTORS AND STABILIZED HOMOLOGY OF SMALL CATEGORIES}

In this section we slightly generalize a result from 7 which relates the stabilization (in the sense of 0.1 ) of a small class of functors to their appropriately stabilized Hochschild-Mitchell homology.

Definition. (See [2].) Let $\mathcal{A}$ be a small category and let $D: \mathcal{A}^{o p} \times \mathcal{A} \rightarrow A b$ be a bifunctor from $\mathcal{A}$ to abelian groups. We let $F_{*}(\mathcal{A} ; D)$ be the simplicial abelian 
group defined by setting

$$
F_{p}(\mathcal{A} ; D)=\bigoplus_{\vec{A} \in N_{p} \mathcal{A}} D\left(A_{1}, A_{0}\right), \quad \vec{A}=A_{1} \stackrel{\alpha_{1}}{\longleftarrow} \cdots \stackrel{\alpha_{p}}{\longleftarrow} A_{0} .
$$

If we represent an element of one component by $\left(g ; \alpha_{1}, \ldots, \alpha_{p}\right)$, then the face and degeneracy operators are given by

$$
\begin{aligned}
& d_{i}\left(g ; \alpha_{1}, \ldots, \alpha_{p}\right)= \begin{cases}\left(D\left(\alpha_{1}, i d\right)(g) ; \alpha_{2}, \ldots, \alpha_{p}\right), & i=0, \\
\left(g ; \ldots, \alpha_{i} \alpha_{i+1}, \ldots \alpha_{p}\right), & 1 \leq i \leq p-1, \\
\left(D\left(i d, \alpha_{p}\right)(g) ; \alpha_{1}, \ldots, \alpha_{p-1}\right), & i=p,\end{cases} \\
& s_{i}\left(g ; \alpha_{1}, \ldots, \alpha_{p}\right)= \begin{cases}\left(g ; \ldots, \alpha_{i}, i d_{A_{i+1}}, \alpha_{i+1}, \ldots\right), & 0 \leq i \leq p-1, \\
\left(g ; \alpha_{1}, \ldots, \alpha_{p}, i d_{A_{0}}\right), & i=p .\end{cases}
\end{aligned}
$$

The homology of $F_{*}(\mathcal{C} ; D)$ is the Hochschild-Mitchell homology of the category $\mathcal{C}$ with coefficients in the bifunctor $D$.

Definition. Let $\mathcal{E}$ be an exact category. A local coefficient system $G$ (at $\mathcal{E}$ ) associates a bifunctor $G_{\mathcal{A}}$ from $\mathcal{A}^{o p} \times \mathcal{A}$ to simplicial abelian groups for each $\mathcal{A} \in \mathcal{S}_{\mathcal{E}}$ such that

(i) $G_{\mathcal{A}}$ is bireduced- $G(0, A)=0=G(A, 0)$ for all $A \in \mathcal{A}$

(ii) $G$ is natural - for every morphism $F: \mathcal{A} \longrightarrow \mathcal{B}$ in $\mathcal{S}_{\mathcal{E}}$, there is a natural transformation of bifunctors $G_{F}: G_{\mathcal{A}} \longrightarrow G_{\mathcal{B}}$ such that $G_{i d}=i d$ and $G_{F \circ F^{\prime}}=G_{F} \circ G_{F^{\prime}}$.

Example. If $M_{1}, \ldots, M_{n}$ are $R$-bimodules, we have a local coefficient system $G\left(M_{1}, \ldots, M_{n}\right)$ at $\mathcal{P}$ given by

$$
G_{\mathcal{A}}\left(A, A^{\prime}\right)=\bigotimes_{i=1}^{n} \operatorname{Hom}_{\mathcal{S}_{I}(\mathcal{A})}\left(\mathcal{S}_{I}(A), \mathcal{S}_{M_{i}}\left(A^{\prime}\right)\right)
$$

Notation. Let $G$ be a local coefficient system for $\mathcal{E}$. By naturality, $F_{\star}\left(\star, G_{\star}\right)$ is a functor from $\mathcal{S}_{\mathcal{E}}$ to simplicial abelian groups. For the purposes of proposition 2.1 below we will simply write $F_{*}\left(S^{(k)}\right)$ for the $k+1$-simplicial abelian group determined by $F_{*}\left(S^{(k)} \mathcal{A} ; G\right)$ when $\mathcal{A}$ and $G$ are clear. Let $\delta$ be the natural transformation given by degeneracies from $F_{0}$ to $F_{*}$.

Proposition (2.1) (similar to 6] for the case $G(R)$ ). Let $G$ be a local coefficient system for $\mathcal{E}$. The natural transformation $\delta\left(S^{(N)}\right)$ from $F_{0}\left(S^{(N)}\right)$ to $F_{*}\left(S^{(N)}\right)$ is $2 N-1$-connected, and hence $\delta^{\text {st }}$ (as in 0.1) is an equivalence.

Proof. More generally, we show that for all $n \in \mathbf{N}$, the map from $F_{0} S^{(N)} \mathcal{A}$ to $F_{n} S^{(N)} \mathcal{A}$ given by degeneracies is $2 N-1$-connected, which implies the result by a standard spectral sequence argument. Let $c$ be the natural transformation from $F_{n}$ to $F_{0}$ defined by sending $\left(g ; \alpha_{1}, \ldots, \alpha_{n}\right)$ to $\left(G\left(\alpha_{1} \cdots \alpha_{n} ; i d\right)(g)\right)$. Since $c \circ \operatorname{deg}=i d_{F_{0}}$, it suffices to show that $C=\operatorname{deg} \circ c$ agrees with the identity in a $2 N-1$ range when we include $S^{(N)}$ into the picture. In other words, we want to show that the simplicial self map $C$ of $F_{n} S^{(N)} \mathcal{A}$ defined by sending $\left(g ; \alpha_{1}, \ldots, \alpha_{n}\right)$ to $\left(G\left(\alpha_{1} \cdots \alpha_{n} ; i d\right)(g) ; i d_{C_{0}}, \ldots, i d_{C_{0}}\right)$ is $2 N-1$ connected.

To prove this we are going to use the fact that $F_{n} S^{(N)}$ satisfies additivity in a $2 N-1$ range. We construct three natural transformations $T_{i d}, T_{-c}$ and $T_{t}$ 
from $F_{n}$ to $F_{n} S_{2}$, which then assemble to give simplicial maps from $F_{n} S^{(N)} \mathcal{A}$ to $F_{n} S^{(N)} S_{2} \mathcal{A}$. We define $T_{i d}, T_{-c}$ and $T_{t}$ as follows.

Let $\vec{\alpha}=\left(g ; C_{1} \stackrel{\alpha_{1}}{\longleftarrow} \cdots \longleftarrow C_{n} \stackrel{\alpha_{n}}{\longleftarrow} C_{0}\right)$ be an element of $F_{n}(\mathcal{A} ; G)$ and let $\alpha_{i \cdots j}$ be the composite $\alpha_{i} \alpha_{i+1} \cdots \alpha_{j}$. Then

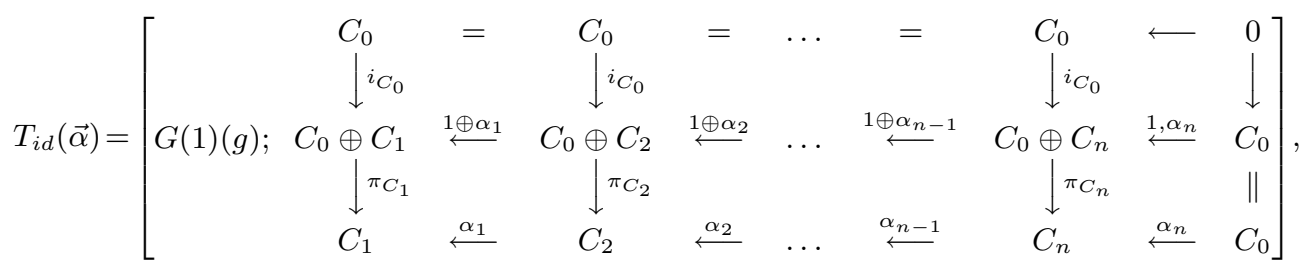

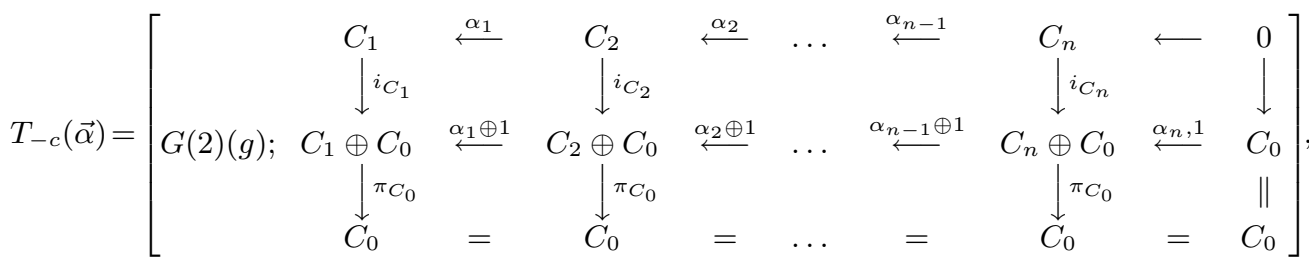

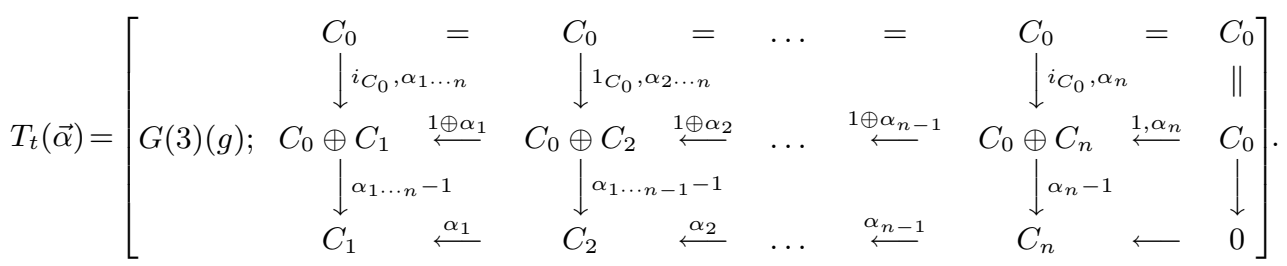

The map $G(1)$ is the natural group homomorphism

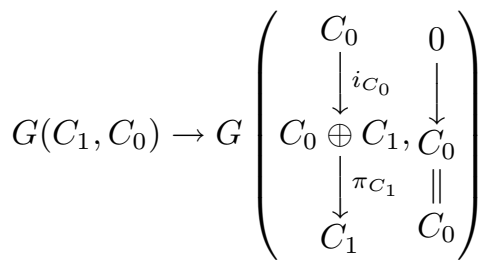

given by the composite $G\left(\pi_{s_{0}\left(C_{1}\right)}, i d\right) \circ G_{s_{0}}$, where $s_{0}: S_{1} \longrightarrow S_{2}$ is the degeneracy map taking $C$ to $0 \rightarrow C=C$ and $\pi_{s_{0}\left(C_{1}\right)}$ is the projection map (of the direct sum)

$$
\left(\begin{array}{c}
C_{0} \\
\underset{C_{0}}{\downarrow} i_{C_{0}} \\
\underset{\downarrow}{\oplus} C_{1} \\
\underset{C_{1}}{\downarrow} \pi_{C_{1}} \\
C_{1}
\end{array}\right) \rightarrow\left(\begin{array}{c}
0 \\
\downarrow \\
C_{1} \\
\| \\
C_{1}
\end{array}\right)
$$


The map $G(2)$ is the natural group homomorphism

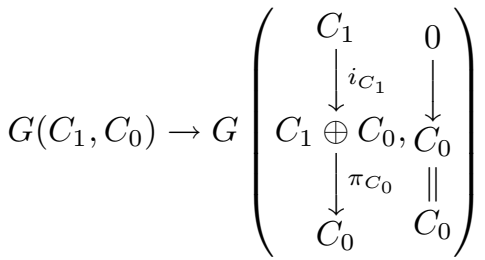

given by the composite $(-1) G\left(\pi_{s_{0}\left(C_{0}\right)}, i d\right) \circ G_{s_{0}} \circ G\left(\alpha_{1 \cdots n}, i d\right)$.

The map $G(3)$ is the difference of two natural maps $G_{1}(3)$ and $G_{2}(3)$

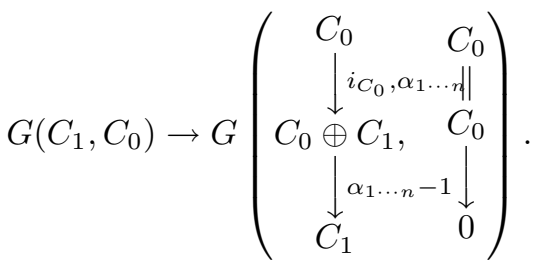

The map $G_{1}(3)$ is given by the composite

$$
G\left(\begin{array}{c}
\alpha_{1 \cdots n} \\
\pi_{C_{1}}, i d \\
0
\end{array}\right) \circ G_{s_{1}}
$$

and the map $G_{2}(3)$ is given by the composite

$$
G\left(\pi_{s_{1}\left(C_{0}\right)}\right) \circ G_{s_{1}} \circ G\left(\alpha_{1 \cdots n}, i d\right) .
$$

Now we note the following relations:

$$
\begin{gathered}
d_{0} T_{i d}=i d, \quad d_{0} T_{-c}=-C, \quad d_{0} T_{t}=0, \\
d_{1} T_{t}=d_{1} T_{i d}+d_{1} T_{-c}, \quad d_{2} T_{i d}=d_{2} T_{-c}=d_{2} T_{t}=0 .
\end{gathered}
$$

By additivity we obtain, in a $2 N-1$ range,

$$
\begin{aligned}
i d-C & =d_{0} T_{i d}+d_{0} T_{-c} \\
& =\left(d_{0} T_{i d}+d_{2} T_{i d}\right)+\left(d_{0} T_{-c}+d_{2} T_{-c}\right) \\
& \simeq d_{1} T_{i d}+d_{1} T_{-c} \\
& =d_{1} T_{t} \\
& \simeq d_{0} T_{t}+d_{2} T_{t} \\
& =0
\end{aligned}
$$

and hence the result.

\section{Computation of the stabilized tensor products}

In this section we compute the stabilization (in the sense of 0.1 ) of $n$-fold tensor product functors. Our second step in this calculation is a generalization of methods used in [5] for the special case when $n=1$. If one is only interested in the rational case, this step can be greatly simplified by appealing to a multi-simplicial argument using complexes similar to Hochschild homology (for categories, as in [13]) — which becomes very reminiscent of techniques in [8], section 4 . 
Let $M_{1}, \ldots, M_{n}$ be fixed $R$-bimodules and let $G\left(M_{1}, \ldots, M_{n}\right)$ (or just $G$ when $M_{1}, \ldots, M_{n}$ are clear) be the local coefficient system of section 2 given by

$$
G(\mathcal{A})\left(A, A^{\prime}\right)=\bigotimes_{i=1}^{n} \operatorname{Hom}_{\mathcal{S}_{I} \mathcal{A}}\left(\mathcal{S}_{I}(A), \mathcal{S}_{M_{i}}\left(A^{\prime}\right)\right)
$$

For $\sigma \in \Sigma_{n}$ (where $\Sigma_{n}$ is the group of permutations on $n$-letters) we let

$$
\sigma_{*}: G\left(M_{1}, \ldots, M_{n}\right) \rightarrow G\left(M_{\sigma(1)}, \ldots, M_{\sigma(n)}\right)
$$

be the evident natural transformation by rearranging the $n$-fold tensor product.

We will simply write $F_{*}^{n}$ for $F_{*}(\mathcal{A} ; G)$ in this section. Thus:

$$
\begin{gathered}
{[p] \longrightarrow F_{p}^{n} \equiv \bigoplus_{\vec{A} \in N_{p} \mathcal{A}} \bigotimes_{i=1}^{n} \operatorname{Hom}_{\mathcal{S}_{I} \mathcal{A}}\left(\mathcal{S}_{I} A_{1}, \mathcal{S}_{M_{i}} A_{0}\right),} \\
\vec{A}=A_{1} \longleftarrow \cdots \longleftarrow A_{p} \longleftarrow A_{0},
\end{gathered}
$$

with the face and degeneracy operators as defined before.

For $\sigma \in \Sigma_{n}$, we let $F^{\sigma} \mathcal{A}$ be the simplicial abelian group:

$$
\begin{gathered}
{[p] \longrightarrow F_{p}^{\sigma} \equiv \bigoplus_{\vec{A} \in N_{p} \mathcal{A}^{n}} \operatorname{Hom}_{\mathcal{S}_{I} \mathcal{A}}\left(\mathcal{S}_{I} A_{1,1}, \mathcal{S}_{M_{1}} A_{0, \sigma(1)}\right)} \\
\otimes \cdots \otimes \operatorname{Hom}_{\mathcal{S}_{I} \mathcal{A}}\left(\mathcal{S}_{I} A_{1, n}, \mathcal{S}_{M_{n}} A_{0, \sigma(n)}\right), \\
\vec{A}=\left\{A_{1, i} \longleftarrow \cdots \longleftarrow A_{n, i} \longleftarrow A_{0, i}\right\}_{i=1}^{n},
\end{gathered}
$$

with face and degeneracy operators like those for $F^{n}$ only being careful about order. We define

$$
F^{\Sigma_{n}}=\bigoplus_{\sigma \in \Sigma_{n}} F^{\sigma}
$$

We define a map of simplicial abelian groups $\psi_{\sigma}: F^{n} \longrightarrow F^{\sigma}$ by taking the indexing nerve to the diagonal of the product and $\alpha \in \bigotimes_{i=1}^{n} \operatorname{Hom}_{\mathcal{S}_{I} \mathcal{A}}\left(\mathcal{S}_{I} A_{1}, \mathcal{S}_{M_{i}} A_{0}\right)$ to itself. We define the natural transformation $\Delta$ from $F^{n}$ to $F^{\Sigma_{n}}$ by $\Delta=\bigoplus \psi_{\sigma}$. There is a natural $\Sigma_{n}$-action on $F^{n}$ given by permuting the $n$-fold tensors. To make $\Delta$ an equivariant map we give $F^{\Sigma_{n}}$ a $\Sigma_{n}$-action by:

(1) permuting the index set $N_{p} \mathcal{A}^{n}$,

(2) rearranging the $n$-fold tensors, and

(3) conjugation on the indexing element of $\Sigma_{n}$. Thus, $\left(\alpha_{1}, \ldots, \alpha_{n} ; \sigma\right) * \tau=\left(\alpha_{\tau(1)}, \ldots, \alpha_{\tau(n)} ; \tau^{-1} \sigma \tau\right)$.

So far, everything is well defined for an arbitrary linear category. Now we define $\phi_{\sigma}: F^{\sigma} \longrightarrow F^{n}$, which is a natural transformation of exact functors which preserve a chosen $\oplus$-action. We define $\phi_{\sigma}$ of $\left(\left\{A_{1, i} \stackrel{\beta_{1, i}}{\longleftarrow} \cdots \stackrel{\beta_{n, i}}{\longleftarrow} A_{0, i}\right\}_{i=1}^{n} ; \gamma_{1} \otimes \cdots \otimes \gamma_{n}\right)$ to be

$$
\left(\bigoplus A_{1, i} \stackrel{\oplus \beta_{1, i}}{\longleftarrow} \cdots \stackrel{\oplus \beta_{n, i}}{\longleftarrow} \bigoplus A_{0, i} ; \tilde{\gamma}_{1} \otimes \cdots \otimes \tilde{\gamma}_{n}\right)
$$

where

$$
\gamma_{j} \in \operatorname{Hom}_{\mathcal{S}_{I} \mathcal{A}}\left(\mathcal{S}_{I} A_{1, j}, \mathcal{S}_{M_{j}} A_{0, \sigma(j)}\right)
$$

and

$$
\tilde{\gamma}_{j} \in \operatorname{Hom}_{\mathcal{S}_{I} \mathcal{A}}\left(\mathcal{S}_{I} \bigoplus A_{1, i}, \mathcal{S}_{M_{j}} \bigoplus A_{0, i}\right)
$$


is the unique morphism (as a natural transformation for all $\gamma_{j}$ ) which is $\gamma_{j}$ and zeros elsewhere (using the identification $\left.\mathcal{S}_{M_{j}} \bigoplus A_{0, i} \cong \bigoplus S_{M_{j}} A_{0, i}\right)$. We define $\phi: F^{\Sigma_{n}} \longrightarrow F^{n}$ by $\phi=\Sigma \phi_{\sigma}$.

Proposition (3.2). The maps $\Delta$ and $\phi$ are homotopy inverses of each other.

Proof. The proof of this proposition is in several steps. Our proof uses three sublemmas. In each sub-lemma we will be constructing semi-simplicial homotopies (they satisfy the simplicial homotopy identities with respect to the face maps, see 11], section 5). Given a semi-simplicial homotopy $\left\{h_{i}\right\}$, one can construct a chain homotopy $H$ by setting $H_{n}=\sum_{i=0}^{n}(-1)^{i} h_{i}$.

Sub-lemma 1.

$$
\psi_{\sigma} \circ \phi_{\tau} \simeq \begin{cases}i d, & \text { if } \sigma=\tau \\ 0, & \text { if } \sigma \neq \tau\end{cases}
$$

We first note that $\psi_{\sigma} \circ \phi_{\tau}$ of $\left(\left\{A_{1, i} \stackrel{\beta_{1, i}}{\longleftarrow} \ldots \stackrel{\beta_{n, i}}{\longleftarrow} A_{0, i}\right\}_{i=1}^{n} ; \gamma_{1} \otimes \cdots \otimes \gamma_{n}\right)$ is

$$
\left(\left\{\bigoplus A_{1, j} \stackrel{\oplus \beta_{1, j}}{\longleftarrow} \cdots \stackrel{\oplus \beta_{n, j}}{\longleftarrow} \bigoplus A_{0, j}\right\}_{i=1}^{n} ; \tilde{\gamma}_{1} \otimes \cdots \otimes \tilde{\gamma}_{n}\right) .
$$

We construct a semi-simplicial homotopy $h_{t}$ as follows. Let $\pi_{u}: \bigoplus_{i=1}^{n} A_{k, i} \longrightarrow A_{k, u}$ be the natural projection. We set $h_{t}(0 \leq t \leq n)$ of $\left(\left\{A_{1, i} \stackrel{\beta_{1, i}}{\longleftarrow} \ldots \stackrel{\beta_{n, i}}{\longleftarrow} A_{0, i}\right\}_{i=1}^{n} ; \gamma_{1} \otimes\right.$ $\left.\cdots \otimes \gamma_{n}\right)$ to be

$$
\left(\left\{A_{1, i} \stackrel{\beta_{1, i}}{\longleftarrow} \ldots \stackrel{\beta_{t-1, i}}{\longleftarrow} A_{t, i} \stackrel{\pi_{i}}{\longleftarrow} \bigoplus_{j=0}^{n} A_{t, j} \stackrel{\oplus \beta_{t, i}}{\longleftarrow} \cdots \stackrel{\oplus \beta_{n, i}}{\longleftarrow} \bigoplus_{j=0}^{n} A_{0, j}\right\}_{i=1}^{n} ; \hat{\gamma}_{1} \otimes \cdots \otimes \hat{\gamma}_{n}\right),
$$

where $\gamma_{i} \in \operatorname{Hom}_{\mathcal{S}_{I} \mathcal{A}}\left(\mathcal{S}_{I} A_{1, i}, \mathcal{S}_{M_{i}} A_{0, \sigma(i)}\right)$ and $\hat{\gamma}_{i} \in \operatorname{Hom}_{\mathcal{S}_{I} \mathcal{A}}\left(\mathcal{S}_{I} A_{1, i}, \mathcal{S}_{M_{i}} \bigoplus A_{0, i}\right)$ is the unique morphism which is $\gamma_{i}$ and zeros elsewhere. One can check that this gives the desired homotopy. In particular, $d_{n+1} h_{n}$ is 0 if $\sigma \neq \tau$ and the identity if $\sigma=\tau$.

Let $\mathcal{M}_{n}$ be the set of all set maps from $\{1, \ldots, n\}$ to itself. For each $\lambda \in \mathcal{M}_{n}$, we define $\theta^{\lambda}$, a simplicial self map of $F^{n} \mathcal{A}$, by sending $\left(A_{1} \stackrel{\beta_{1}}{\longleftarrow} \cdots \stackrel{\beta_{n}}{\longleftarrow} A_{0} ; \gamma_{1} \otimes \cdots \otimes \gamma_{n}\right)$ to

$$
\left(A_{1}^{\oplus n} \stackrel{\beta_{1}^{\oplus n}}{\longleftarrow} \cdots \stackrel{\beta_{n}^{\oplus n}}{\longleftarrow} A_{0}^{\oplus n} ; \tilde{\gamma}_{1, \lambda(1)} \otimes \cdots \otimes \tilde{\gamma}_{n, \lambda(n)}\right),
$$

where $\tilde{\gamma}_{i, j} \in \operatorname{Hom}_{\mathcal{S}_{I} \mathcal{A}}\left(\mathcal{S}_{I} A_{1}^{\oplus n}, \mathcal{S}_{M_{i}} A_{0}^{\oplus n}\right)$ is $\gamma_{i}$ in the $(i, j)$ position and zeros elsewhere. We let $\Theta=\sum_{\lambda \in \mathcal{M}_{n}} \theta^{\lambda}$.

Sub-lemma 2. $\Theta \simeq i d_{F^{n}}$.

We define the semi-simplicial homotopy $\left\{h_{i}\right\}$ as follows. Let $\delta: A \longrightarrow A^{\oplus n}$ be the diagonal map. We set $h_{i}$ of $\left(A_{1} \stackrel{\beta_{1}}{\longleftarrow} \cdots \stackrel{\beta_{n}}{\longleftarrow} A_{0} ; \gamma_{1} \otimes \cdots \otimes \gamma_{n}\right)$ to be

$$
\left(A_{1}^{\oplus n} \stackrel{\beta_{1 n}^{\oplus n}}{\longleftarrow} \cdots \stackrel{\beta^{\oplus n}}{\longleftarrow} A_{i+1}^{\oplus n} \stackrel{\delta}{\longleftarrow} A_{i+1} \stackrel{\beta_{i+1}}{\longleftarrow} \cdots \stackrel{\beta_{n}}{\longleftarrow} A_{0} ; \bar{\gamma}_{1} \otimes \bar{\gamma}_{2} \otimes \cdots \otimes \bar{\gamma}_{n}\right),
$$

where $\bar{\gamma}_{j} \in \operatorname{Hom}_{\mathcal{S}_{I} \mathcal{A}}\left(\mathcal{S}_{I} \bigoplus A_{1}, \mathcal{S}_{M_{j}} A_{0}\right)$ is $\gamma_{j} \circ \mathcal{S}_{I} \pi_{j}$, where $\pi_{j}$ is the natural projection of $\bigoplus A_{1}$ onto its $j$-th coordinate. It is clear that $d_{0} h_{0}$ is the identity. Similarly, $d_{n+1} h_{n}=\Theta$, since $d_{n+1} h_{n}$ is

$$
\left(\left(A_{1}^{\oplus n} \stackrel{\beta_{1}^{\oplus n}}{\longleftarrow} \cdots \stackrel{\beta_{n}^{\oplus n}}{\longleftarrow} A_{0}^{\oplus n} ; \sum_{j_{1}=1}^{n} \tilde{\gamma}_{1, j_{1}} \otimes \cdots \otimes \sum_{j_{n}=1}^{n} \tilde{\gamma}_{n, j_{n}}\right)\right.
$$


and by multilinearity

$$
\begin{aligned}
\sum_{j_{1}=1}^{n} \tilde{\gamma}_{1, j_{1}} \otimes \cdots \otimes \sum_{j_{n}=1}^{n} \tilde{\gamma}_{n, j_{n}} & =\sum_{j_{1}, \ldots, j_{n}=1}^{n} \tilde{\gamma}_{1, j_{1}} \otimes \cdots \otimes \tilde{\gamma}_{n, j_{n}} \\
& =\sum_{\lambda \in \mathcal{M}_{n}} \tilde{\gamma}_{1, \lambda(1)} \otimes \cdots \otimes \tilde{\gamma}_{n, \lambda(n)} .
\end{aligned}
$$

Sub-lemma 3. $\theta^{\lambda} \simeq 0$ if $\lambda$ is not surjective.

For $\lambda \in \mathcal{M}_{n}$, we let $[A]_{\lambda} \in \operatorname{Hom}_{\mathcal{A}}\left(A^{\oplus n}, A^{\oplus n}\right)$ be the morphism which is the identity in positions $(i, \lambda(i))$ for all $1 \leq i \leq n$ and zeros elsewhere. Suppose $\lambda$ is not surjective and let $k \notin \operatorname{Image}(\lambda)$. We define a semi-simplicial homotopy by setting $h_{i}$ of $\left(A_{1} \stackrel{\beta_{1}}{\longleftarrow} \cdots \stackrel{\beta_{n}}{\longleftarrow} A_{0} ; \gamma_{1} \otimes \cdots \otimes \gamma_{n}\right)$ to be

$$
\left(A_{1}^{\oplus n} \stackrel{\beta_{1}^{\oplus n}}{\longleftarrow} \cdots \stackrel{\beta_{i}^{\oplus n}}{\longleftarrow} A_{i+1}^{\oplus n} \stackrel{\left[A_{i+1}\right]_{\lambda}}{\longleftarrow} A_{i+1}^{\oplus n} \stackrel{\beta_{i+1}^{\oplus n}}{\longleftarrow} \cdots \stackrel{\beta_{n}^{\oplus n}}{\longleftarrow} A_{0}^{\oplus n} ; \tilde{\gamma}_{1,1} \otimes \cdots \otimes \tilde{\gamma}_{n, n}\right) .
$$

Then $d_{0} h_{0}=\theta^{\lambda}$ and $d_{n+1} h_{n}=0$, since $k \neq\{1, \ldots, n\}$ implies that $\left[A_{1}\right]_{\lambda} \circ \tilde{\gamma}_{k, k}=0$.

Proof of proposition 3.2. The composite map $\Delta \circ \phi$ is equal to $\Sigma \psi_{\sigma} \circ \phi_{\tau}$, which is homotopic to $\sum i d_{\sigma}$ by sub-lemma 1 . The composite map $\phi \circ \Delta$ is equal to $\sum \phi_{\sigma} \circ \psi_{\sigma}=\sum \theta^{\sigma}$, which by sub-lemma 3 is homotopic to $\Theta$, which by sub-lemma 2 is homotopic to the identity.

We now define a new construction $F^{\mathcal{S}_{n}}$. Let $\mathcal{S}_{n}$ be the subset of $\Sigma_{n}$ consisting of cycles of length $n$. We note that $\mathcal{S}_{n}$ is an invariant subset by conjugation. Also,

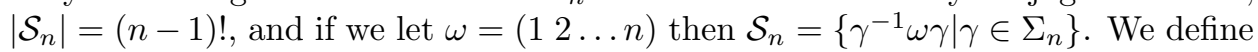
$F^{\mathcal{S}_{n}}$ to be the subsimplicial abelian group of $F^{\Sigma_{n}}$ determined by

$$
F^{\mathcal{S}_{n}}=\bigoplus_{\sigma \in \mathcal{S}_{n}} F^{\sigma}
$$

We note that $F^{\mathcal{S}_{n}}$ is a $\Sigma_{n}$-invariant subsimplicial abelian group of $F^{\Sigma_{n}}$.

Lemma (3.3). If $\mathcal{A}_{*}$ is an $n$-reduced simplicial exact category, then $F^{\mathcal{S}_{n}} \mathcal{A}_{*} \longrightarrow$ $F^{\Sigma_{n}} \mathcal{A}_{*}$ is a $2 n-1$ connected map.

Proof. If $\sigma$ can be written as the product of two disjoint cycles $\tau \circ \gamma$, then $F^{\sigma} \cong$ $F^{\tau} \otimes F^{\gamma}$, and hence $F^{\sigma} \mathcal{A}_{*}$ would be at least $2 n$ connected. Thus, $F^{\Sigma_{n}} \mathcal{A}_{*}=$ $F^{\mathcal{S}_{n}} \mathcal{A}_{*} \oplus$ (terms $2 n$ connected and higher).

Theorem (3.4). Using the notation of 0.1 , if $M_{1}=M_{2}=\cdots=M_{n}$ then $\left(F^{n}\right)^{\text {st }}$ is naturally $\Sigma_{n}$ equivalent to $\mathbf{Z}\left[\Sigma_{n}\right] \otimes_{\mathbf{Z}\left[C_{n}\right]}\left(F^{\omega}\right)^{s t}$.

Proof. By 3.2, $\Delta: F^{n} \longrightarrow F^{\Sigma_{n}}$ is a homotopy equivalence for all $\mathcal{A} \in \mathcal{S}_{\mathcal{P}}$, and hence by 3.3 we obtain

$$
\left(F^{n}\right)^{s t}=\lim _{k \rightarrow \infty} F^{n} S^{k}[-k] \stackrel{\simeq}{\longrightarrow} \lim _{k \rightarrow \infty} F^{\Sigma_{n}} S^{k}[-k] \stackrel{\simeq}{\longleftarrow} \lim _{k \rightarrow \infty} F^{\mathcal{S}_{n}} S^{k}[-k] .
$$

Now we note that the natural map $\mathbf{Z}\left[\Sigma_{n}\right] \otimes_{\mathbf{Z}\left[C_{n}\right]} F^{\omega} \longrightarrow F^{\mathcal{S}_{n}}$ given by sending $(\sigma \otimes x)$ to $\sigma_{*}(x)$ (as defined in 3.1) is a $\Sigma_{n}$ equivariant isomorphism of simplicial abelian groups.

We now slightly generalize theorem 1.4 of [5] so that we may rewrite $\left(F^{\omega}\right)^{s t}$. 
Definition. Given a linear functor $G$ from $\mathcal{A}$ to $\mathcal{B}$, we define the "twisted" product category $\mathcal{A}_{G} \mathcal{B}$ as follows. We set $\operatorname{Obj}\left(\mathcal{A}_{G} \mathcal{B}\right)$ to be $\operatorname{Obj}(\mathcal{A}) \times \operatorname{Obj}(\mathcal{B})$ and

$$
\operatorname{Hom}_{\mathcal{A}_{G} \mathcal{B}}\left((A, B),\left(A^{\prime}, B^{\prime}\right)\right)=\operatorname{Hom}_{\mathcal{A}}\left(A, A^{\prime}\right) \oplus \operatorname{Hom}_{\mathcal{B}}\left(B, B^{\prime}\right) \oplus \operatorname{Hom}_{\mathcal{B}}\left(G(A), B^{\prime}\right)
$$

with composition defined by $(f, g, h) \circ\left(f^{\prime}, g^{\prime}, h^{\prime}\right)=\left(f \circ f^{\prime}, g \circ g^{\prime}, h \circ G\left(f^{\prime}\right)+g \circ h^{\prime}\right)$.

For fixed $X=(A, B), Y=\left(A^{\prime}, B^{\prime}\right) \in \mathcal{A}_{G} \mathcal{B}$, let $C_{*}$ be the simplicial abelian group

$$
\begin{gathered}
{[p] \longrightarrow C_{p} \equiv \bigoplus_{\vec{C} \in N_{p} \mathcal{A}_{G} \mathcal{B}} \operatorname{Hom}_{\mathcal{A}_{G} \mathcal{B}}\left(C_{1}, X\right) \otimes \operatorname{Hom}_{\mathcal{A}_{G} \mathcal{B}}\left(Y, C_{0}\right),} \\
\vec{C}=C_{1} \longleftarrow \cdots \longleftarrow C_{p} \longleftarrow C_{0}
\end{gathered}
$$

with the face and degeneracies given like those for $F^{n}$. We will represent an arbitrary generating element of $C_{n}$ by $\left(\beta_{0} \otimes \alpha_{0} ; \alpha_{1}, \ldots, \alpha_{n}\right)$. We let $F^{(1)}(\mathcal{A})$ be the simplicial functor

$$
\begin{array}{r}
{[p] \mapsto F_{p}^{(1)}(\mathcal{A})=\bigoplus_{\vec{A} \in N_{p} \mathcal{A}} \operatorname{Hom}_{\mathcal{A}}\left(A_{1}, A\right) \otimes \operatorname{Hom}_{\mathcal{A}}\left(A^{\prime}, A_{0}\right),} \\
\vec{A}=A_{1} \longleftarrow \cdots \longleftarrow A_{p} \longleftarrow A_{0},
\end{array}
$$

and let $F^{(1)}(\mathcal{B})$ be the simplicial functor

$$
\begin{array}{r}
{[p] \mapsto F_{p}^{(1)}(\mathcal{B})=\bigoplus_{\vec{B} \in N_{p} \mathcal{B}} \operatorname{Hom}_{\mathcal{B}}\left(B_{1}, B\right) \otimes \operatorname{Hom}_{\mathcal{B}}\left(B^{\prime}, B_{0}\right),} \\
\vec{B}=B_{1} \longleftarrow \cdots \longleftarrow B_{p} \longleftarrow B_{0}
\end{array}
$$

with the face and degeneracies given like those for $F^{n}$.

Proposition (3.5). The functor from $\mathcal{A}_{G} \mathcal{B}$ to $\mathcal{A} \times \mathcal{B}$ which is the identity on objects (and sends $(f, g, h)$ to $f \times g$ ) produces a homotopy equivalence from $C_{*}$ to $F^{(1)}(\mathcal{A}) \times F^{(1)}(\mathcal{B})$.

Proof. We will (once again) be defining several chain homotopies which arise from semi-simplicial homotopies (they satisfy the simplicial homotopy identities with respect to the face maps; see [11, section 5). Given a semi-simplicial homotopy $\left\{h_{i}\right\}$, one can construct a chain homotopy $H$ by setting $H_{n}=\sum_{i=0}^{n}(-1)^{i} h_{i}$.

First reduction: The subcomplex of $C_{*}$ generated by elements of the form $\left(\beta_{0} \otimes\right.$ $\left.\alpha_{0} ; \alpha_{1}, \ldots, \alpha_{n}\right)$ such that $\alpha_{0}=\left(0,0, h_{0}\right)$ and $\beta_{0}=\left(0,0, h_{0}^{\prime}\right)$ is acyclic.

$\underline{\text { Proof. }}$. We let $\alpha_{i}=\left(f_{i}, g_{i}, h_{i}\right)$ and define a semi-simplicial homotopy from the identity to 0 as follows:

$$
\begin{aligned}
& h_{i}\left(\left(0,0, h_{0}^{\prime}\right) \otimes\left(0,0, h_{0}\right) ; \alpha_{1}, \ldots, \alpha_{n}\right) \\
& \quad=\left(\left(0,0, h_{0}^{\prime}\right) \otimes\left(0,0, h_{0}\right) ;\left(0, g_{1}, 0\right), \ldots,\left(0, g_{i}, 0\right),\left(0, i d_{B_{i+1}}, 0\right), \alpha_{i+1}, \ldots, \alpha_{n}\right) .
\end{aligned}
$$


Now we quotient $C_{*}$ by this acyclic subcomplex to get a new complex $\tilde{C}_{*}$. We will write a generating element of this complex as $\left(\left(f_{0}^{\prime}, g_{0}^{\prime}, \star^{\prime}\right) \otimes\left(f_{0}, g_{0}, \star\right) ; \alpha_{1}, \ldots, \alpha_{n}\right)$. The complex $\tilde{C}_{*}$ splits into a sum of four subcomplexes: $\tilde{C}_{*}=A A_{*} \oplus A B_{*} \oplus B A_{*} \oplus$ $B B_{*}$, where

$$
\begin{aligned}
& A A_{n} \text { is generated by }\left(\left(f_{0}^{\prime}, 0, \star^{\prime}\right) \otimes\left(f_{0}, 0, \star\right) ; \alpha_{1}, \ldots, \alpha_{n}\right), \\
& A B_{n} \text { is generated by }\left(\left(f_{0}^{\prime}, 0, \star^{\prime}\right) \otimes\left(0, g_{0}, \star\right) ; \alpha_{1}, \ldots, \alpha_{n}\right), \\
& B A_{n} \text { is generated by }\left(\left(0, g_{0}^{\prime}, \star^{\prime}\right) \otimes\left(f_{0}, 0, \star\right) ; \alpha_{1}, \ldots, \alpha_{n}\right), \\
& B B_{n} \text { is generated by }\left(\left(0, g_{0}^{\prime}, \star^{\prime}\right) \otimes\left(0, g_{0}, \star\right) ; \alpha_{1}, \ldots, \alpha_{n}\right) .
\end{aligned}
$$

Second reduction: The projection from $A A_{*} \oplus A B_{*}$ to $F^{(1)} \mathcal{A}$ generated by sending $\left(\left(f_{0}^{\prime}, 0, \star^{\prime}\right) \otimes\left(f_{0}, 0, \star\right) ; \alpha_{1}, \ldots, \alpha_{n}\right)$ to $\left(f_{0}^{\prime} \otimes f_{0} ; f_{1}, \ldots, f_{n}\right)$ and $A B_{*}$ to 0 is a homotopy equivalence.

$\underline{\text { Proof. }}$. Choose elements $a$ of $\mathcal{A}$ and $b$ of $\mathcal{B}$. The projection has a section defined by sending $\left(f_{0}^{\prime} \otimes f_{0} ; f_{1}, \ldots, f_{n}\right)$ to the equivalence class containing

$$
\left(\left(f_{0}^{\prime}, 0_{b}, \star\right) \otimes\left(f_{0}, 0_{b}, \star\right) ;\left(f_{1}, 0_{b}, 0\right), \ldots,\left(f_{n}, 0_{b}, 0\right)\right),
$$

where we let $0_{b}$ denote the zero endomorphism of $b$. A simplicial homotopy from the identity to the composite can be defined by sending the class of

$$
\left(\left(f_{0}^{\prime}, 0, \star^{\prime}\right) \otimes\left(f_{0}, 0, \star\right) ; \alpha_{1}, \ldots, \alpha_{n}\right) \oplus\left(\left(\hat{f}_{0}^{\prime}, 0, \star^{\prime}\right) \otimes\left(0, \hat{g}_{0}, \star\right) ; \hat{\alpha}_{1}, \ldots, \hat{\alpha}_{n}\right)
$$

by $h_{i}$ to the class of

$$
\begin{aligned}
& \left(\left(f_{0}^{\prime}, 0, \star^{\prime}\right) \otimes\left(f_{0}, 0, \star\right) ;\left(f_{1}, 0_{b}, 0\right), \ldots,\left(f_{i}, 0_{b}, 0\right),\left(i d_{A_{i+1}}, 0,0\right), \alpha_{i+1}, \ldots, \alpha_{n}\right) \\
& \oplus \\
& \left(\left(\hat{f}_{0}^{\prime}, 0, \star^{\prime}\right) \otimes\left(0, \hat{g}_{0}, \star\right) ;\left(0_{a}, \hat{g}_{1}, 0\right), \ldots,\left(0_{a}, \hat{g}_{i}, 0\right),\left(0, i d_{\hat{B}_{i+1}}, 0\right), \hat{\alpha}_{i+1}, \ldots, \hat{\alpha}_{n}\right) .
\end{aligned}
$$

Third reduction: The projection from $B B_{*} \oplus B A_{*}$ to $F^{(1)} \mathcal{B}$ generated by sending a generating element $\left(\left(0, g_{0}^{\prime}, \star^{\prime}\right) \otimes\left(0, g_{0}, \star\right) ; \alpha_{1}, \ldots, \alpha_{n}\right)$ to $\left(g_{0}^{\prime} \otimes g_{0} ; g_{1}, \ldots, g_{n}\right)$ and $B A_{*}$ to 0 is a homotopy equivalence.

$\underline{\text { Proof. }}$. Choose some element $a$ of $\mathcal{A}$. The projection has a section defined by sending $\left(g_{0} ; g_{1}, \ldots, g_{n}\right)$ to the equivalence class containing

$$
\left(\left(0_{a}, g_{0}^{\prime}, \star^{\prime}\right) \otimes\left(0_{a}, g_{0}, \star\right) ;\left(0_{a}, g_{1}, 0\right), \ldots,\left(0_{a}, g_{n}, 0\right)\right),
$$

where we let $0_{a}$ denote the zero endomorphism of $a$. A simplicial homotopy from the composite to the identity can be defined by sending the class of

$$
\left.\left(\left(0, g_{0}^{\prime}, \star^{\prime}\right) \otimes\left(0, g_{0}, \star\right) ; \alpha_{1}, \ldots, \alpha_{n}\right) \oplus\left(0, \hat{g}_{0}^{\prime}, \star^{\prime}\right) \otimes\left(\hat{f}_{0}, 0, \star\right) ; \hat{\alpha}_{1}, \ldots, \hat{\alpha}_{n}\right)
$$

by $h_{i}$ to the class of

$$
\begin{gathered}
\left(\left(0, g_{0}^{\prime}, \star^{\prime}\right) \otimes\left(0, g_{0}, \star\right) ; \alpha_{1}, \ldots, \alpha_{i},\left(0, i d_{B_{i+1}}, 0\right),\left(0_{a}, g_{i+1}, 0\right), \ldots,\left(0_{a}, g_{n}, 0\right)\right) \\
\oplus \\
\left.\left(0, \hat{g}_{0}^{\prime}, \star^{\prime}\right) \otimes\left(\hat{f}_{0}, 0, \star\right) ; \hat{\alpha}_{1}, \ldots, \hat{\alpha}_{i},\left(0, i d_{\hat{B}_{i+1}}, 0\right),\left(0_{a}, \hat{g}_{i+1}, 0\right), \ldots,\left(0_{a}, \hat{g}_{n}, 0\right)\right) .
\end{gathered}
$$


We have constructed a diagram of complexes

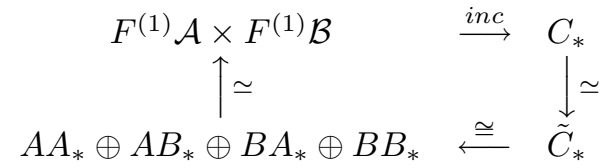

with the maps up and down quasi-isomorphisms by reductions 1-3 above. Since the composite around the square is the identity on $F^{(1)} \mathcal{A} \times F^{(1)} \mathcal{B}$, we see that inc is a quasi-isomorphism. Since the inclusion inc is a section to our map, we are done.

Corollary (3.6). For $f: \mathcal{A} \rightarrow \mathcal{B}$ a linear functor, the natural map

$$
F^{\omega}(\mathcal{A}) \oplus F^{\omega}(\mathcal{B}) \rightarrow F^{\omega}\left(\mathcal{A}_{f} \mathcal{B}\right)
$$

is an equivalence.

Proof. We can consider $F^{\omega}$ as the diagonal of an $n$-simplicial abelian group. By the Eilenberg-Zilber theorem it suffices to show that the map is an equivalence on the associate $n$-dimensional complexes. We can factor the map into $n$-steps, where we pass from the product category to the twisted category in each simplicial dimension one at a time separately. Each of these maps is levelwise an example of proposition 3.5 except for the tensor of a module. However, since proposition 3.5 was obtained by chain homotopies, it remains true after tensoring with a fixed module. Thus, each of the $n$ maps is an equivalence by the realization lemma (or standard spectral sequence arguments), and we are finished.

Corollary (3.7). For any $n$ we have $\Omega F^{\omega} S \stackrel{\simeq}{\longrightarrow}\left(F^{\omega}\right)^{\text {st }}$, and if the exact category $\mathcal{C}$ is split (all cofibrations have a retract), then $F^{\omega} \mathcal{C} \stackrel{\simeq}{\longrightarrow} \Omega F^{\omega}$ S.C.

The proof is exactly as in section 1 of [5]).

Corollary (3.8). If $M_{1}=M_{2}=\cdots=M_{n}$, then the functor $\left(F^{n}\right)^{\text {st }}$ is naturally $\Sigma_{n}$ equivalent (in the weak sense-that is, connected by a chain of $\Sigma_{n}$-equivariant maps which are also equivalences non-equivariantly) to $\Omega\left[\mathbf{Z}\left[\Sigma_{n}\right] \otimes_{\mathbf{Z}\left[C_{n}\right]}\left(F^{\omega} S.\right)\right]$, and in particular

$$
\left(F^{n}\right)^{s t}(\mathcal{P}) \sim_{\Sigma_{n}} \mathbf{Z}\left[\Sigma_{n}\right] \otimes_{\mathbf{Z}\left[C_{n}\right]} F^{\omega}(\mathcal{P}) .
$$

We will now rewrite our functors $F^{\omega}$ in terms of Hochschild homology when $\mathbf{Q} \subseteq R$. More generally, one should use Mac Lane homology to rewrite these, which we will do in the next section.

There is a natural simplicial map $\tau$ from $F^{\omega}(\mathcal{P})$ to $H H\left(R^{\otimes n},\left(\bigotimes_{j=1}^{n} M_{i}\right)_{\tau}\right)$ which is $\mathbf{Z}\left[C_{n}\right]$-equivariant when the $M_{i}$ 's are equal given in simplicial dimension $p-1$ by the composite shown in Figure 1, which is given by the evaluation maps $\mathbf{Z}[G] \rightarrow G$ which take $\sum z_{i}\left[g_{i}\right]$ to $\sum z_{i} \cdot g_{i}$ for any abelian group $G$, and where the map "trace" is the Dennis trace map (as used in [13]).

Proposition (3.9). If $\mathbf{Q} \subseteq R$, then the simplicial map $\tau$ from $F^{\omega} \mathcal{P}$ to

$$
H H\left(R^{\otimes n},\left(\bigotimes_{j=1}^{n} M_{i}\right)\right),
$$

is an equivalence which is $C_{n}$-equivariant when the $M_{i}$ 's are equal. 


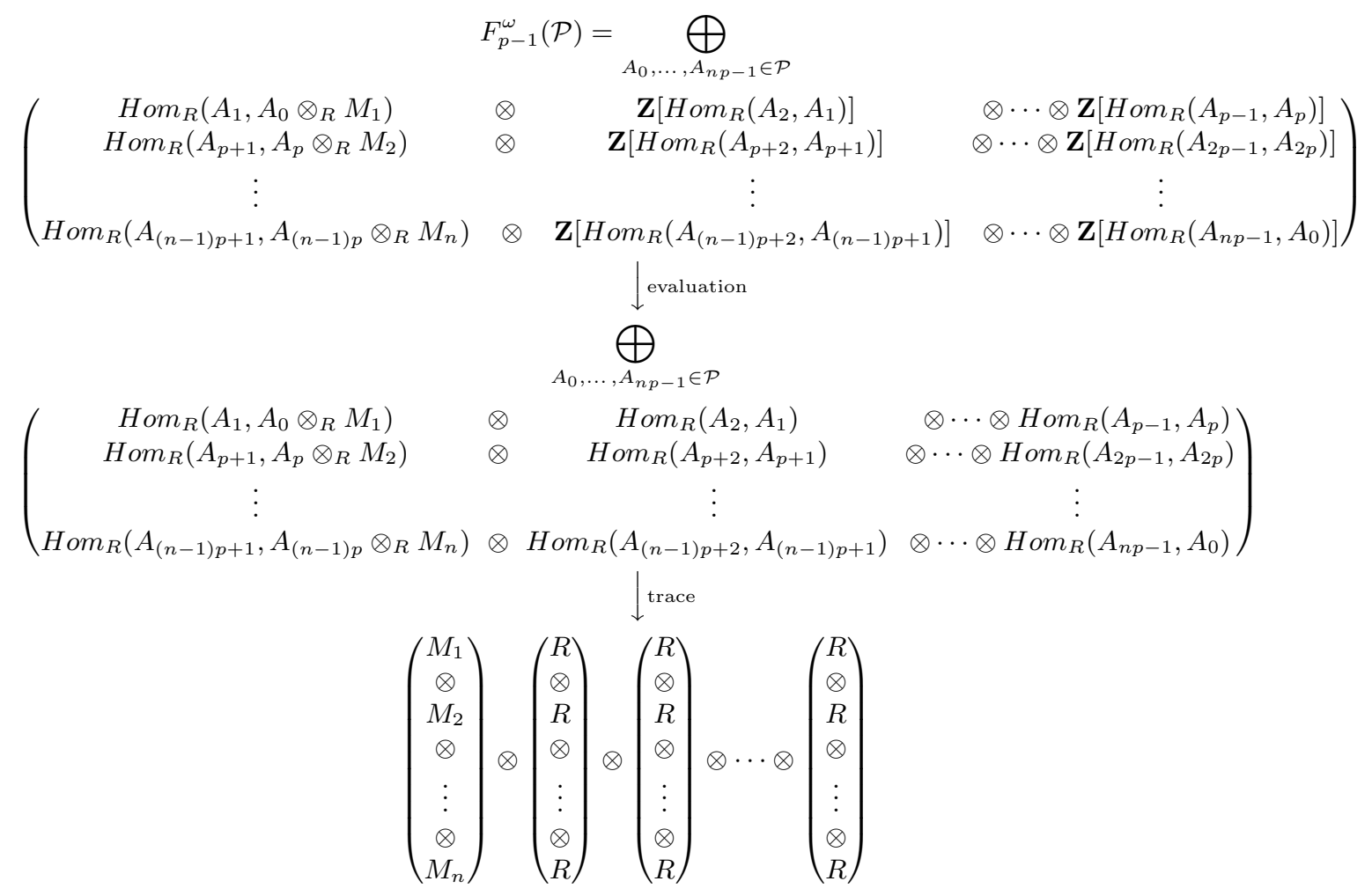

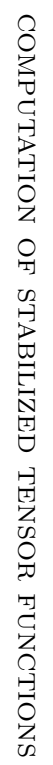

Figure 1. 
Proof. The map $\tau$ is the diagonal of an $n$-dimensional simplicial map we call $\tau^{\prime}$. By the Eilenberg-Zilber theorem, it suffices to show $\tau^{\prime}$ is an equivalence. The map $\tau^{\prime}$ can be decomposed as the composite of $n$ maps of $n$-simplicial abelian groups, where one applies the map " $\tau$ " to one dimension at a time. Levelwise, each of these maps is a rational equivalence by 1.4.3 and 1.4.8 of [7], and hence by the realization lemma (or a standard spectral sequence argument) each of the $n$-composites is a rational equivalence, and so $\tau^{\prime}$ is a rational equivalence.

\section{Relation to Mac Lane homology}

In this section we rewrite our computation from section 3 in terms of Mac Lane homology. We will assume the reader is familiar with Mac Lane's $Q$-construction (e.g. [9] or [10]).

We can rewrite $F_{*}$ as

$$
\begin{aligned}
F_{n}(\mathcal{C} ; D)=\bigoplus_{A_{0}, \ldots, A_{n} \in \mathcal{C}} D\left(A_{1}, A_{0}\right) \otimes_{\mathbf{z}} \mathbf{Z}\left[\operatorname{Hom}_{\mathcal{C}}\left(A_{2}, A_{1}\right)\right] \\
\otimes_{\mathbf{z}} \cdots \otimes_{\mathbf{z}} \mathbf{Z}\left[\operatorname{Hom}_{\mathcal{C}}\left(A_{0}, A_{n}\right)\right],
\end{aligned}
$$

where $\mathbf{Z}[X]$ is the free abelian group generated by a pointed set $X$.

For any abelian group, we have natural maps $\mathbf{Z}[G] \stackrel{\beta}{\longrightarrow} Q_{*}(G) \stackrel{\gamma}{\longrightarrow} G$, where $Q_{*}(G)$ is Mac Lane's $Q$-construction (an explicit chain construction whose homology is the stable homology of the group $G$ ). We also recall that there is a natural map $Q_{*}(G) \otimes_{\mathbf{Z}} Q_{*}\left(G^{\prime}\right) \stackrel{\mu}{\longrightarrow} Q_{*}\left(G \otimes \mathbf{z} G^{\prime}\right)$ which can be used to give $Q_{*}(R)$ the structure of a differential graded algebra when $R$ is a ring and such that $\gamma_{R}$ becomes a map of differential graded algebras. We note that the natural map $Z[G] \otimes Z\left[G^{\prime}\right] \cong Z\left[G \times G^{\prime}\right] \rightarrow Z\left[G \otimes \mathbf{z} G^{\prime}\right]$ commutes with $\mu$ via $\beta$.

Let $D$ be a bi-additive functor. Set $Q_{*}(\mathcal{C} ; D)$ to be the simplicial chain complex defined by

$$
\begin{aligned}
Q_{n}(\mathcal{C} ; D)=\bigoplus_{A_{0}, \ldots, A_{n} \in \mathcal{C}} D\left(A_{1}, A_{0}\right) & \otimes_{\mathbf{z}} Q_{*}\left(\operatorname{Hom}_{\mathcal{C}}\left(A_{2}, A_{1}\right)\right) \\
& \otimes_{\mathbf{z}} \cdots \otimes_{\mathbf{z}} Q_{*}\left(\operatorname{Hom}_{\mathcal{C}}\left(A_{0}, A_{n}\right)\right)
\end{aligned}
$$

with simplicial structure maps like those of $F_{*}$ using the natural maps $\gamma$ for $d_{0}$ and $d_{n+1}$ and $\mu$ otherwise. By 1.4 .8 of $\left[7, Q_{*}(\mathcal{C} ; D)\right.$ is naturally equivalent to $T H(\mathcal{C} ; D)$. Using $\beta$, we obtain a natural transformation of simplicial objects

$$
F_{*}(\mathcal{C} ; D) \stackrel{\beta}{\longrightarrow} Q_{*}(\mathcal{C} ; D),
$$

which is an equivalence for $\mathcal{C}$ a split exact category by the main result of [16]. We note that one can also obtain this result by modifying the proof of proposition 2.1 using $F_{0}(\mathcal{C} ; D)=Q_{0}(\mathcal{C} ; D)$.

We identify $R$ with the subcategory of $\mathcal{P}$ generated by a free module of rank 1 . By inclusion of subcategories, we obtain a map of simplicial objects

$$
Q_{*}\left(R ;\left.D\right|_{R}\right) \stackrel{i}{\longrightarrow} Q_{*}(\mathcal{P} ; D),
$$

which is a natural equivalence by 2.1 .5 of [7]. 
Theorem (4.1). Let $M$ be an R-bimodule and let $G(M, \ldots, M)$ be the the local coefficient system on $\mathcal{P}$ as defined in section 2. Then

$$
G(M, \ldots, M)^{s t}(\mathcal{P}) \sim_{\Sigma_{n}} \mathbf{Z}\left[\Sigma_{n}\right] \otimes_{\mathbf{Z}\left[C_{n}\right]} H H\left(Q_{*}(R)^{\otimes n} ; M_{\tau}^{\otimes n}\right),
$$

where $H H$ is the Hochschild homology complex for $Q_{*}(R)^{\otimes n}$ acting on the bimodule by

$$
\begin{gathered}
\left(m_{1} \otimes \cdots \otimes m_{n}\right) *\left(q_{1} \otimes \cdots \otimes q_{n}\right)=\left(m_{1} q_{1} \otimes m_{2} q_{2} \otimes \cdots \otimes m_{n} q_{n}\right), \\
\left(q_{1} \otimes \cdots \otimes q_{n}\right) *\left(m_{1} \otimes \cdots \otimes m_{n}\right)=\left(q_{n} m_{1} \otimes q_{1} m_{2} \otimes \cdots \otimes q_{n-1} m_{n}\right),
\end{gathered}
$$

and $C_{n}$ is the cyclic group of $n$ elements, which acts by cyclic permutations - the equivalence is weakly $\Sigma_{n}$-equivariant.

Proof. By corollary 3.8 , it suffices to show that $F^{\omega}$ is weakly equivalent to

$$
H H\left(Q_{*}(R)^{\otimes n} ; M_{1} \otimes \mathbf{z} \cdots \otimes \mathbf{z} M_{n}\right)
$$

in a $C_{n}$-equivariant manner when the $M_{i}$ 's are equal. Just as we defined $Q_{*}$ for $F_{*}$, we can define $Q_{*}^{\omega}$ with (appropriately $C_{n}$ equivariant) maps

$$
F^{\omega}(\mathcal{P}) \stackrel{\beta^{\prime}}{\longrightarrow} Q^{\omega}(\mathcal{P}) \stackrel{i^{\prime}}{\longleftarrow} Q^{\omega}(R) .
$$

Since $Q^{\omega}(R)$ is isomorphic to $H H\left(Q_{*}(R)^{\otimes n} ; M_{1} \otimes_{\mathbf{z}} \cdots \otimes_{\mathbf{z}} M_{n}\right)$, we simply need to show that both $\beta^{\prime}$ and $i^{\prime}$ are equivalences. However, both these maps are the diagonal maps of $n$-fold multi-simplicial maps, and these $n$-fold multi-simplicial maps are $n$-fold composite maps, each of which is an equivalence by repeated applications of $(*)$ and $(* *)$ above.

\section{REFERENCES}

[1] J. F. Adams, Stable homotopy and generalised homology, The University of Chicago Press, Chicago (1974). MR 53:6534

[2] H. Baues and G. Wirsching, Cohomology of small categories, J. Pure Appl. Algebra 38 (1985), 187-211. MR 87g:18013

[3] M. Bökstedt, W. C. Hsiang and I. Madsen, The cyclotomic trace and algebraic K-theory of spaces, Invent. Math. 111 (1993), 463-539. MR 94g:55011

[4] A. Dold and D. Puppe, Homologie nicht-additiver Functoren, Anwendungen, Ann. Inst. Fourier 11 (1961), 201-312. MR 27:186

[5] B. Dundas and R. McCarthy, Stable K-theory and topological Hochschild homology, Annals of Math. 140 (1994), 685-701. MR 96e:19005a

[6] B. Dundas and R. McCarthy, Erratum to Stable K-theory and topological Hochschild homology, Annals of Math. 142 (1995), 425-426. MR 96e:19005b

[7] B. Dundas and R. McCarthy, Topological Hochschild homology of ring functors and exact categories, J. Pure Appl. Alg. 109 (1996), 231-294. MR 97i:19001

[8] T. Goodwillie, Relative algebraic K-theory and cyclic homology, Ann. of Math. 121 (1985), 383-407. MR 88b:18008

[9] M. Jibladze and T. Pirashvili, Cohomology of Algebraic Theories, Journal of Algebra, 137 (1991), 253-296. MR 92f:18005

[10] S. Mac Lane, Homologie des anneaux et des modules, CBRM, Colloque de topologie algebrique, Louvain (1956), 55-80. MR 20:892

[11] P. May, Simplicial objects in algebraic topology, D. Van Nostrand Co. (1967). MR 36:5942 
[12] R. McCarthy, On fundamental theorems of algebraic K-theory, Topology 32 (1993), 325-328. MR 94e:19002

[13] R. McCarthy, The cyclic homology of an exact category, J. Pure Appl. Alg. 93 (1994), 251296. MR 95b:19002

[14] R. McCarthy, A chain complex for the spectrum homology of the algebraic K-theory of an exact category, Algebraic $K$-theory (Toronto, 1996), Fields Institute Comm., vol. 16, Amer. Math. Soc., Providence, RI, (1997), 199-219. MR 98k:19004

[15] R. McCarthy, Relative algebraic K-theory and topological cyclic homology, Acta Math., 179 (1997), 197-222. MR 99e:19006

[16] T. Pirashvili and F. Waldhausen, Mac Lane homology and topological Hochschild homology, J. Pure Appl. Algebra 82 (1992), 81-98. MR 93k:16016

[17] F. Waldhausen, Algebraic K-theory of spaces, Springer Lecture Notes in Math. 1126 (1985), 318-419. MR 86m:18011

[18] G. Whitehead, Elements of Homotopy Theory, Springer-Verlag, Graduate Texts in Mathematics 61 (1978). MR 80b:55001

Department of Mathematics, University of Illinois, Urbana, Illinois 61801

E-mail address: randy@math.uiuc.edu 